\title{
CONDITIONS FOR A MODULE TO BE INJECTIVE AND SOME APPLICATIONS TO HOPF ALGEBRA DUALITY
}

\author{
IAN M. MUSSON
}

(Communicated by Lance W. Small)

\begin{abstract}
We present some simple conditions for a module to be injective in certain situations. Injective modules over bialgebras often have the additional structure of module algebras, and our results can be used to give some explicit examples. In particular, we construct such a module algebra for the quantum group $O_{q}(\mathrm{SL}(2))$. Our results can also be used to describe the Hopf algebra duals of $O_{q}(\mathrm{SL}(2))$ and related Hopf algebras.
\end{abstract}

\section{INTRODUCTION}

In this paper we present some easily checked conditions for a module to be injective and give some applications to Hopf algebra duality. We refer to [McR] and $[\mathrm{A}]$ for unexplained terminology.

We denote the injective hull of a (right) module $A$ over a ring $S$ by $E_{S}(A)$. The first situation we consider is where $R$ is a subring of a Noetherian ring $S$ such that $S=R \oplus I$ for an ideal $I$ of $S$ with the AR (Artin-Rees) property. If $A$ is an injective $R$-module, we can regard $A$ as an $S$-module via the isomorphism $S \cong R / I$. In this case we give an explicit description of $E_{S}(A)$ in the corollary of $\S 1.1$.

Now suppose $\sigma$ is an automorphism of the Noetherian ring $R$, and let $S=R[y ; \sigma]$ be the skew polynomial ring, that is, the ring of polynomials in $y$ subject to the relations $r y=y \sigma(r)$ for $r \in R$. Note that $y$ is a normal element of $S$, and so $I=y S$ has the AR property by [McR, 4.1.10]. Clearly $S=R \oplus I$, so the last paragraph applies in this situation.

Motivated by this example we next consider the case where $S$ has a normal element $x$, but the ideal $I=x S$ is not necessarily complemented by a subring. In this case for an injective $(S / I)$-module $A$ we do not have such an explicit description of $E_{S}(A)$, but we give instead in the theorem in $\$ 2.1$ a test for a right $S$-module to be isomorphic to $E_{S}(A)$. This test can be used inductively if $I$ is a polynormal ideal.

Received by the editors December 22, 1992 and, in revised form, June 1, 1993; presented at a meeting of the American Mathematical Society in South Bend, IN, March 15-16, 1991 (meeting No. 864, Abstract 864-16-104).

1991 Mathematics Subject Classification. Primary 16D50, 16W30.

Research partially supported by National Security Agency grant $91 \mathrm{H} 0017$. 
Part of the motivation for our study of injective modules comes from Hopf algebra duality. Here we are concerned with Hopf algebras $H$ which satisfy the following conditions:

(1) Every finite-dimensional simple $H$-module is one dimensional.

(2) The augmentation ideal $m$ of $H$ is polynormal.

Examples of such Hopf algebras include the coordinate ring $O_{q}(\mathrm{SL}(n))$ of quantum $\operatorname{SL}(n)$.

For $f \in H^{*}$ and $s \in H$, we denote the value of $s$ under $f$ by $\langle f \mid s\rangle$. We regard $H^{0} \subseteq H^{*}$ as right $H$-modules by defining $\langle f \cdot r \mid s\rangle=\langle f \mid r s\rangle$ for $f \in H^{*}$, $r, s \in H$.

We review some general results from [CM].

Proposition. Let $H$ be a Noetherian Hopf algebra satisfying conditions (1), (2) above, and let $C=H^{0}$. Then:

(a) The module $E=E_{H}(H / m)$ is a locally finite-dimensional $H$-module.

(b) The dual $C=H^{0}$ contains a copy of $E$, and we have as right $H$-modules $C \cong E \otimes C_{o}$ where $C_{o}$ is the coradical of $C$.

These results are illustrated explicitly here for the case $H=O_{q}(P)$ where $P$ is a "Borel subgroup" of $\mathrm{SL}(2)$ in $\S 3.3$ and for the case $H=O_{q}(\mathrm{SL}(2))$ in [CM, §4].

In many cases $E_{H}(H / m)$ can be embedded as a submodule algebra in $H^{0}$. For example, if $H$ is commutative and Noetherian, then $E_{H}(H / m)$ is isomorphic as an $H$-module to the hyperalgebra $H^{\prime}=\left\{f \in H^{*} \mid f\left(m^{n}\right)=0\right.$ for some $n\}$. This is well known and follows from the corollary in $\S 1.1$. However, we show that if $H=O_{q}(\mathrm{SL}(2))$, then $E_{H}(H / m)$ is not isomorphic to a submodule algebra of $H^{0}$.

In this connection the following result from [CM, Proposition 1.7 and Theorem 3.3] is also relevant.

Theorem. Let $H$ be a bialgebra over an algebraically closed field, and let $E$ be the unique maximal locally finite-dimensional submodule of $E_{H}(H / m)$. Then:

(1) $E$ can be given the structure of an $H$-module algebra.

(2) If $H$ is cocommutative, then $E$ is isomorphic to a submodule algebra of $H^{0}$.

Special cases of the theorem are given in [L] and [M].

The significance of the theorem is that it gives an approach to the study of finite-dimensional $H$-modules. Indeed, if $W$ is any (locally) finite-dimensional $H$-module, then by [CM, Lemma 1.3] $W$ is isomorphic to a submodule of $\operatorname{soc}(W) \otimes E$, where $\operatorname{soc}(W)$ is the socle of $W$. Thus the study of finitedimensional $H$-modules reduces in some sense to the study of the simple modules and the study of $E$. Now if $E$ is a module algebra, its module structure is determined by the module action on the algebra generators of $E$. Furthermore, if (as is often the case) $E$ is a finitely generated algebra, these generators are contained in a finite-dimensional submodule of $E$.

We illustrate these ideas by giving an explicit construction for $E_{H}(H / m)$ as a module algebra when $H=O_{q}(\mathrm{SL}(2))$.

If $H=U_{q}(\mathrm{sl}(n))$ where $q$ is not a root of unity and the characteristic of the ground field is not 2, the Hopf algebra dual $H^{0}$ is described in [T, Theorem 
5.8]; see also [APW, §12] for a related result. A basic fact that is used here is the complete reducibility of finite-dimensional $H$-modules. Thus $H^{0}$ can be calculated from the coefficient functions of finite-dimensional simple modules.

If $H=O_{q}(\mathrm{SL}(n))$, this method is no longer available. However, the methods introduced in this paper and in [CM] may still be used to calculate $H^{0}$.

Results on Hopf algebra duality have various applications in the theory of quantum groups. For example, they are used in the construction of the Drinfeld double $[\mathrm{D}, \mathrm{R}]$ and in the determination of the primitive ideals in $C_{q}[G][\mathrm{HL}]$.

\section{Preliminary Results and eXamples}

1.1. If $I$ is an ideal of a ring $S, R=S / I$, and $A$ is an injective $R$-module, we wish to find an explicit description of $E_{S}(A)$ in certain cases. The question of what constitutes an "explicit description" raises issues in mathematical logic which we do not address here; see [K, 3.1] for some discussion.

In a special case there is a functorial solution to our problem. If $A$ is an $R$ module, we denote the product of elements $a \in A, r \in R$, by $a \cdot r$ to distinguish it from other products that will occur later. If $A$ is an $R$-module and $R$ a subring of $S$, then $\operatorname{Hom}_{R}(S, A)$ is an $S$-module via the rule $(f \cdot s)(t)=f(s t)$ for $s, t \in S$ and $f \in \operatorname{Hom}_{R}(S, A)$. If $B$ is an $S$-module, we set $\Gamma_{I}(B)=$ $\left\{b \in B \mid b \cdot I^{n}=0\right.$ for some $\left.n\right\}$.

The first results are well known. The proofs given in [SV, Lemma 2.13] and [H, Lemma III.3.2] may be easily adapted.

Proposition. (i) If $R$ is a subring of $S$ and $E$ is an injective R-module, then $\operatorname{Hom}_{R}(S, E)$ is an injective $S$-module.

(ii) If $I$ is an ideal of the Noetherian ring $S$ with the AR property and $E$ is an injective $S$-module, then $\Gamma_{I}(E)$ is an injective $S$-module.

Corollary. Suppose that $R$ is a subring of the Noetherian ring $S$ and that $S=$ $R \oplus I$ where $I$ is an ideal with the AR property. If $A$ is an injective $R$-module, then $\Gamma_{I}\left(\operatorname{Hom}_{R}(S, A)\right) \cong E_{S}(A)$.

Proof. The proposition shows that $\Gamma_{I}\left(\operatorname{Hom}_{R}(S, A)\right)$ is injective. We note that $A \cong \operatorname{Hom}_{R}(R, A) \cong \operatorname{ann}_{\mathrm{Hom}_{R}(S, A)} I$ which is a submodule of $\Gamma_{I}\left(\operatorname{Hom}_{R}(S, A)\right)$. Given $f \in \Gamma_{I}\left(\operatorname{Hom}_{R}(S, A)\right), f \neq 0$, choose $n$ so that $f\left(I^{n}\right) \neq 0=f\left(I^{n+1}\right)$. There exists $x \in I^{n}$ such that $f(x) \neq 0$, and we have $f \cdot x \in \operatorname{ann}_{\mathrm{Hom}}(\mathrm{S}, \mathrm{A}) I$. Hence, $\Gamma_{I}\left(\operatorname{Hom}_{R}(S, A)\right)$ is an essential extension of $\operatorname{ann}_{\mathrm{Hom}_{R}(\mathrm{~S}, \mathrm{~A})} I$, and this gives the result.

1.2. Now let $\sigma$ be an automorphism of the Noetherian ring $R, S=R[y ; \sigma]$ the skew polynomial ring, and $I=y S$.

For an injective $R$-module $A$, we give a description of $E_{S}(A)$. This extends the construction given by Northcott $[\mathrm{N}]$ of the injective hull of a simple module over a polynomial ring using "inverse polynomials".

As an $R$-module we have $S=\bigoplus_{n \geq 0} R y^{n}$. Let $A Y_{n}=\left\{a Y_{n} \mid a \in A\right\}$ be an isomorphic copy of $A$ as an abelian group, and define a new action of $R$ on $A Y_{n}$ by setting $a Y_{n} \cdot r=a \cdot \sigma^{n}(r) Y_{n}$, for $a \in A, r \in R$. We make the direct sum $A^{+}=\bigoplus_{n \geq 0} A Y_{n}$ into an $S$-module by $a Y_{n} \cdot y=a Y_{n-1}$ for $n \geq 1$ and $a Y_{0} \cdot y=0$. Then we have an isomorphism of $S$-modules $\Gamma_{I}\left(\operatorname{Hom}_{S}(R, A)\right) \rightarrow$ $A^{+}$sending $f$ to $\sum f\left(y^{n}\right) Y_{n}$.

Corollary. If $A$ is an injective $R$-module, then $A^{+} \cong E_{S}(A)$. 


\section{A TEST FOR INJECTIVITY}

2.1. Let $x_{1}, \ldots, x_{n}$ be a normalizing sequence in the Noetherian ring $S$, and set $M_{i}=\sum_{j \leq i} x_{j} S$. In many situations we can show injectivity of a right $S$ module $E$ by showing by reverse induction on $i$ that $\operatorname{ann}_{E} M_{i}$ is an injective $\left(S / M_{i}\right)$-module. This strategy essentially reduces the problem to the case where $n=1, M=x S, x$ is a normal element, and $\operatorname{ann}_{E} M$ is an injective $(S / M)$ module.

We say that an $S$-module $E$ is divisible by $x \in S$ provided whenever $e^{\prime}$ is an element of $E$ such that $\mathrm{r}$-ann $(x) \subseteq \operatorname{ann}_{S}\left(e^{\prime}\right)$, there is an element $e$ of $E$ such that $e x=e^{\prime}$. It is well known that any injective module is divisible by every element of $S$.

If $E$ is an injective module over a ring $S$, then $\operatorname{ann}_{E} I$ is an injective $(S / I)$ module for any ideal of $S$ [SV, Proposition 2.27].

In certain situations these two conditions can be used to show injectivity.

Theorem. Let $x$ be a normal element in a Noetherian ring $S, M=x S$, and $E$ a right $S$-module such that $\operatorname{ann}_{E} M$ is an essential submodule of $E$. Then $E$ is injective if and only if

(1) $\operatorname{ann}_{E} M$ is an injective $(S / M)$-module, and

(2) $E$ is divisible by $x$.

2.2. Lemma. Let $M$ be an ideal in a Noetherian ring $S$ with the AR property, and let $E$ be an $S$-module such that $\operatorname{ann}_{E} M$ is essential in $E$. Then $E$ is injective provided $\operatorname{ann}_{E} M^{n}$ is an injective $\left(S / M^{n}\right)$-module for all $n$.

Proof. Let $I$ be a right ideal of $S$ and $f: I \rightarrow E$ a module homomorphism. Since $f(I)$ is a finitely generated submodule of $E$, we have $f(I) M^{n}=0$ for some $n$ by the AR property. Again by the AR property we can find $m \geq n$ such that $I \cap M^{m} \subseteq I M^{n}$. Hence, $f\left(I \cap M^{m}\right)=0$ and $f$ induces a map $g: I /\left(I \cap M^{m}\right) \rightarrow \operatorname{ann}_{E} M^{m}$ which extends to a map $\bar{g}: S / M^{m} \rightarrow \operatorname{ann}_{E} M^{m}$ by hypothesis. Composing $\bar{g}$ with the natural map $S \rightarrow S / M^{m}$ we obtain a map extending $f$.

2.3. Proof of the Theorem. Suppose conditions (1) and (2) of the theorem in $\S 2.1$ hold. By Lemma 2.2 it suffices to show that $\operatorname{ann}_{E} M^{n}$ is an injective $\left(S / M^{n}\right)$-module for all $n$. We use induction on $n$. The case $n=1$ is assumed. Suppose that $\operatorname{ann}_{E} M^{n}$ is an injective $\left(S / M^{n}\right)$-module, and set $P=\{s \in S \mid x s \in$ $\left.M^{n+1}\right\}$. Then $M^{n+1} \subseteq M^{n} \subseteq P$, so

$$
\operatorname{ann}_{E} P \subseteq \operatorname{ann}_{E} M^{n} \subseteq \operatorname{ann}_{E} M^{n+1} .
$$

By [SV, Proposition 2.27], $\operatorname{ann}_{E} P$ is an injective $(S / P)$-module. We next establish a divisibility condition for the $\left(S / M^{n+1}\right)$-module $\operatorname{ann}_{E} M^{n+1}$. Namely, suppose $e^{\prime} \in \operatorname{ann}_{E} P$ is given. Then $x s=0$ implies $s \in P$, so $e^{\prime} s=0$. Therefore, there exists $e \in E$ such that $e x=e^{\prime}$. Also $e M^{n+1} \subseteq e x M^{n}=e^{\prime} M^{n}=0$, so $e \in \operatorname{ann}_{E} M^{n+1}$.

In order to show that $\operatorname{ann}_{E} M^{n+1}$ is an injective $\left(S / M^{n+1}\right)$-module we can now assume that $M^{n+1}=0$ and use this modified divisibility condition.

Suppose $I$ is a right ideal of $S$ and $f: I \rightarrow E$ is a module homomorphism. Set $J=\{s \in S \mid x s \in I\}$. Define a map $g: J \rightarrow E$ by $g(s)=f(x s)$. If $s \in J \cap P$, then $x s \in x P=0$, so $g(s)=0$. Since $J P \subseteq J \cap P$, we have 
$g(J) P=g(J P)=0$. Hence, $g$ induces a map $h: J /(J \cap P) \rightarrow \operatorname{ann}_{E} P$ which extends to a map $\bar{h}: S / P \rightarrow \operatorname{ann}_{E} P$ by hypothesis. We can view $\bar{h}$ as a map from $S$ to $\operatorname{ann}_{E} P$ with $\bar{h}(s)=g(s)$ for $s \in J$. By the divisibility condition established above we can choose an $e \in E$ with $e x=\bar{h}(1)$. Now define $k: S \rightarrow E$ by $k(s)=e s$ for $s \in S$. If $s \in I \cap x S$, write $s=x r$; then $r \in J$ and $k(s)=e x r=\bar{h}(r)=g(r)=f(s)$. Hence, $k-f$ induces a map $k-f: I /(I \cap x S) \rightarrow \operatorname{ann}_{E} x S$. By assumption $\operatorname{ann}_{E} x S$ is an injective $(S / x S)$-module. Thus, $k-f$ extends to a map $l: S / x S \rightarrow \operatorname{ann}_{E} x S$, and we view $l$ as a map from $S$ to $E$. Then it is easily seen that $\bar{f}=k-l: S \rightarrow E$ extends the original map $f$.

\section{INJECTIVE MODULES OVER BIALGEBRAS}

3.1. Let $H$ be an algebra over a field $F$, and $H^{*}=\operatorname{Hom}_{F}(H, F)$. If $\varepsilon: H \rightarrow$ $F$ is an algebra homomorphism with $\operatorname{Ker} \varepsilon=m$, then $F \varepsilon$ is an $H$-submodule of $H^{*}$ isomorphic to $H / m$. It is well known that $H^{*}$ is an injective $H$-module and hence $H^{*}$ contains a copy of $E_{H}(H / m)$. We give an explicit embedding of $E_{H}(H / m)$ into $H^{*}$.

Suppose that $D$ is any essential extension of the $H$-module $F \varepsilon$ with basis $\left\{v_{\alpha} \mid \alpha \in A\right\}$ where $v_{0}=\varepsilon$. If $d \in D$, we define $g_{d} \in H^{*}$ by $\left\langle g_{d} \mid s\right\rangle=a_{0}$ where $d \cdot s=\sum a_{\alpha} v_{\alpha}$. The map $\phi: D \rightarrow H^{*}$ defined by $\phi(d)=g_{d}$ is a homomorphism of $H$-modules. Moreover if $d \neq 0$, then since $d \cdot H$ is an essential extension of $F \varepsilon$, we have $d \cdot s=\varepsilon$ for some $d \in H$, and hence $\left\langle g_{d} \mid s\right\rangle \neq 0$. Hence, $\phi$ is one-to-one. Note also that if $D$ is finite dimensional, then $D I=0$ for some ideal $I$ of finite codimension in $H$ and so the image of $\varphi$ is contained in $H^{0}$.

3.2. Now suppose $H$ is a bialgebra over $F$ with comultiplication $\Delta$ and counit $\varepsilon$. Then $H^{*}$ becomes an algebra with identity $1_{H^{*}}=\varepsilon$, whose product is determined by the equation

$$
\langle f g \mid s\rangle=\sum_{(s)}\left\langle f \mid s_{(1)}\right\rangle\left\langle g \mid s_{(2)}\right\rangle
$$

for $f, g \in H^{*}, s \in H$. Here we use the "sigma notation"

$$
\Delta(s)=\sum_{(s)} s_{(1)} \otimes s_{(2)}
$$

Now $H^{0}$ is a subalgebra of $H^{*}$ and, in fact, is a bialgebra. The applications of our work on injective modules to the study of $H^{0}$ are based on the next result.

Lemma. If $T$ is a right $H$-submodule of $H^{0}$, then $T=H^{0}$ if and only if

(1) $\operatorname{soc}(T)=\operatorname{soc}\left(H^{0}\right)$, and

(2) $T$ is injective in the category of locally finite-dimensional right $\mathrm{H}$ modules.

Proof. By [G, (1.5a)] $T$ is injective as a left $H^{0}$-comodule. However, the category of left $H^{0}$-comodules is equivalent to the category of locally finite dimensional right $H$-modules, [A, p. 126]. Now if conditions (1) and (2) hold, then $T$ is a direct summand of $H$ as a right $H$-module by injectivity. Since $\operatorname{soc}(T)=\operatorname{soc}\left(H^{0}\right)$, we must have $T=H^{0}$. 
3.3. Now consider the "quantum group" $O_{q}(\mathrm{SL}(2))$ (see [Ma]), that is, $O_{q}(\mathrm{SL}(2))=F[a, b, c, d]$ with relations

$$
\begin{gathered}
b a=q a b, \quad c a=q a c, \quad d b=q b d, \quad d c=q c d, \\
b c=c b, \quad a d-d a=\left(q^{-1}-q\right) b c, \quad a d-q^{-1} b c=1 .
\end{gathered}
$$

We can make $O_{q}(\mathrm{SL}(2))$ into a Hopf algebra by defining

$$
\begin{gathered}
\Delta(a)=a \otimes a+b \otimes c, \quad \Delta(b)=a \otimes b+b \otimes d, \\
\Delta(c)=c \otimes a+d \otimes c, \quad \Delta(d)=c \otimes b+d \otimes d ; \\
\varepsilon(a)=\varepsilon(d)=1, \quad \varepsilon(b)=\varepsilon(c)=0 ; \\
S(a)=d, \quad S(d)=a, \quad S(b)=-q b, \quad S(c)=-q^{-1} c .
\end{gathered}
$$

The normal element $c$ generates a Hopf ideal in $O_{q}(\mathrm{SL}(2))$, and the factor algebra with respect to this ideal may be regarded as the coordinate ring $O_{q}(P)$ of the "quantum Borel subgroup" $P$ of SL(2).

For the remainder of this section let $H=O_{q}(P)$ and $m=\operatorname{Ker} \varepsilon$. We give descriptions of $E_{H}(H / m)$ and $H^{0}$. We assume the basefield $F$ has characteristic zero.

Denoting the images of $d, b$ in $H$ by $x, y$, respectively, we have $H=$ $F\left[x^{ \pm 1}, y\right]$ with the relation $x y=q y x$. Thus, $H=R[y ; \sigma]$, a skew polynomial ring where $R=F\left[x, x^{-1}\right]$ and $\sigma$ is the automorphism of $R$ given by $\sigma(x)=$ $q x$.

The comultiplication, counit, and antipode of $H$ are given by

$$
\begin{array}{cc}
\Delta(x)=x \otimes x, & \Delta(y)=y \otimes x+x^{-1} \otimes y, \\
\varepsilon(x)=1, \quad \varepsilon(y)=0, \quad S(x)=x^{-1}, \quad S(y)=-q y .
\end{array}
$$

Now $H$ has a basis consisting of monomials $y^{j} x^{k}$, so we may define elements $X$ and $Y_{n}$ of $H^{*}$ by

$$
\left\langle X \mid y^{j} x^{k}\right\rangle=k \delta_{0, j} \quad \text { and } \quad\left\langle Y_{n} \mid y^{j} x^{k}\right\rangle=\delta_{n, j} .
$$

Under the action of $H$ on $H^{*}$ given in the introduction we find that

$$
X \cdot x=X+1, \quad X \cdot y=0, \quad Y_{n} \cdot x=q^{n} Y_{n}, Y_{n} \cdot y=Y_{n-1} \quad \text { if } n>0 .
$$

From these relations $X, Y_{n}$ generate finite-dimensional submodules of $H^{*}$, and so $X, Y_{n} \in C=H^{0}$. Note that $Y_{0}=1 \in C$.

For $\lambda \in F^{*}$ we define grouplike elements $g_{\lambda} \in C$ with $\operatorname{Ker} g_{\lambda}=y H+$ $(x-\lambda) H$ and let $G=\left\{g_{\lambda} \mid \lambda \in F^{*}\right\}$.

We denote the subspace of $C$ spanned by the elements $Y_{n}$ (resp. $X^{m} Y_{n}$ ) by $D$ (resp. $E$ ).

Proposition. With the above notation:

(1) $D$ and $E$ are subalgebras of $C=H^{0}$. Moreover, $D$ is an algebra of $q$-divided powers and $E \cong D[X ; \delta]$ a skew polynomial ring defined by $g X-X g=\delta(g)$ where $\delta$ is the derivation of $D$ given by $\delta\left(Y_{n}\right)=2 n Y_{n}$.

(2) $E$ is a right $H$-submodule of $C$ and is isomorphic to $E_{H}(H / m)$.

(3) $C$ is a crossed product $C=E^{*} G$. 
Proof. Define for nonnegative integers $m \leq n$

$$
\begin{aligned}
& {[0]_{q}=1, \quad[n]_{q}=\left(q^{n}-q^{-n}\right) /\left(q-q^{-1}\right) \quad \text { if } n \geq 1,} \\
& {[n] !_{q}=[n]_{q}[n-1]_{q} \cdots[0]_{q},\left[\begin{array}{c}
n \\
m
\end{array}\right]_{q}=\frac{[n] !_{q}}{[m] !_{q}[n-m] !_{q}} .}
\end{aligned}
$$

A routine induction shows that

$$
\Delta\left(y^{j}\right)=\sum_{i=0}^{j}\left[\begin{array}{l}
j \\
i
\end{array}\right]_{q} y^{i} x^{i-j} \otimes y^{j-i} x^{i}
$$

Hence,

$$
\Delta\left(y^{j} x^{k}\right)=\sum_{i=0}^{j}\left[\begin{array}{l}
j \\
i
\end{array}\right]_{q} y^{i} x^{i-j+k} \otimes y^{j-i} x^{i+k} .
$$

It follows by induction on $m$ that $\left\langle X^{m} \mid y^{j} x^{k}\right\rangle=\delta_{o j} k^{m}$, and then we find

$$
\left\langle X^{m} Y_{n} \mid y^{j} x^{k}\right\rangle=\sum_{i=0}^{j}\left[\begin{array}{l}
j \\
i
\end{array}\right]_{q}\left\langle X^{m} \mid y^{i} x^{i-j+k}\right\rangle\left\langle Y_{n} \mid y^{j-i} x^{i+k}\right\rangle=\delta_{n j}(k-n)^{m} .
$$

Using similar calculations we can check that the following relations hold in $C$ :

$$
\begin{gathered}
Y_{n} X-X Y_{n}=2 n Y_{n}, \quad Y_{m} Y_{n}=\left[\begin{array}{c}
m+n \\
m
\end{array}\right]_{q} Y_{m+n}, \\
Y_{n} g_{\lambda}=\lambda^{2 n} g_{\lambda} Y_{n}, \quad g_{\lambda} X=X g_{\lambda}, \quad g_{\lambda} g_{\mu}=g_{\lambda \mu} .
\end{gathered}
$$

We claim that the elements $X^{m} Y_{n}$ of $C$ are linearly independent. Indeed if $\sum_{m, n} \alpha_{m n} X^{m} Y_{n}=0$, then evaluating on $y^{j} x^{k}$ we obtain $\sum_{m} \alpha_{m j}(k-j)^{m}=0$ for all $j, k$, so the claim follows using vanderMonde determinants. Part (1) now follows.

Using the fact that $C$ is an $H$-module algebra we easily see that

$$
\begin{gathered}
\left(X^{m} Y_{n}\right) \cdot x=q^{n}(X+1)^{m} Y_{n}=X^{m} \cdot \sigma^{n}(x) Y_{n}, \\
\left(X^{m} Y_{n}\right) \cdot y=(X-1)^{m} Y_{n-1} .
\end{gathered}
$$

We have $\operatorname{ann}_{E}(y H)=F[X]$, and this is an injective $(H / y H)$-module. Also $E$ is divisible by $y$. Thus by the theorem in $\S 2.1, E \cong E_{H}(H / m)$, proving (2).

As a right $H$-module $C \cong E \otimes F G$ by the proposition in the introduction. We sketch the proof. For $\lambda \in F^{*}, E g_{\lambda} \cong E \otimes F g_{\lambda}$ as a right $H$-module, and by [CM, 1.3] this module is the injective hull of $F g_{\lambda}$. Now the sum $T=\sum E g_{\lambda}$ is direct since the socles of the modules $E g_{\lambda}$ and $E g_{\mu}$ are nonisomorphic when $\lambda \neq \mu$. Thus, $T$ is injective as a right $H$-module. We have $\operatorname{soc}(T)=\operatorname{soc}(C)=$ $\sum F g_{\lambda}$, and thus $T=C$ by the lemma in $\S 3.2$. From the relations established above $C$ is a crossed product $C=E^{*} G$.

To complete the description of $C$ as a Hopf algebra we compute $\Delta_{C}, \varepsilon_{C}$, and $S_{C}$. Set $K=g_{q}$. We have

$$
\begin{gathered}
\Delta_{C}(X)=X \otimes 1+1 \otimes X, \\
\Delta_{C}\left(Y_{n}\right)=\sum_{i=0}^{n} q^{i(n-i)} K^{(n-i)} Y_{i} \otimes Y_{n-i}, \\
\varepsilon_{C}(X)=0, \quad \varepsilon_{C}\left(Y_{n}\right)=\delta_{n 0}, \quad S_{C}(X)=-X .
\end{gathered}
$$


For example, the formula for $\Delta_{C}\left(Y_{n}\right)$ follows by computing

$$
\left\langle Y_{n} \mid y^{j} x^{k} y^{r} x^{s}\right\rangle=\sum_{i=0}^{n} q^{i(n-i)}\left\langle K^{(n-i)} Y_{i} \mid y^{j} x^{k}\right\rangle\left\langle Y_{n-i} \mid y^{r} x^{s}\right\rangle=\delta_{n, j+r} q^{k r}
$$

Finally $S_{C}\left(Y_{n}\right)$ may be found inductively using

$$
0=\varepsilon_{C}\left(Y_{n}\right)=\sum_{i=0}^{n} q^{i(n-i)} K^{(n-i)} Y_{i} S\left(Y_{n-i}\right) \text { if } n>0 .
$$

Remark. If the field $F$ has positive characteristic, there is a similar structural result using divided powers of $X$.

3.4. Now let $H=O_{q}(\operatorname{SL}(2))$ and $m=\operatorname{Ker} \varepsilon$. We show that $E=E_{H}(H / m)$ can be given the structure of an $H$-module algebra. However, $E$ is not isomorphic to a submodule algebra of $H^{0}$. We assume for simplicity that $q \in F-\{0\}$ is not a root of unity and that $F$ has characteristic 0 .

To construct $E$ as a module algebra we use the following result.

Lemma. Let $H$ be a bialgebra and $V$ a (right) $H$-module. Then the action of $H$ on $V$ extends in a unique way to an action on the tensor algebra $T=T(V)$, making $T$ into an $H$-module algebra. Suppose that $H$ is generated as an algebra by a subspace $W$ such that $\Delta(W) \subseteq W \otimes W$. If $J$ is the two-sided ideal of $T$ generated by elements $\left\{x_{\gamma} \mid \gamma \in \Gamma\right\}$, then $T / J$ inherits the structure of an $H$-module algebra provided $x_{\gamma} \cdot w \in J$, for all $\gamma \in \Gamma$ and all $w \in W$.

Proof. It is well known that $T(V)$ has a unique $H$-module algebra structure. It is enough to show that $J$ is an $H$-submodule of $T(V)$. This follows since for $r, s \in T$ and $w \in W$ we have

$$
\left(r x_{\gamma} s\right) \cdot w=\sum_{(w)} r \cdot w_{(1)} x_{\gamma} \cdot w_{(2)} s \cdot w_{(3)} \in J .
$$

Now let $V$ be the $H=O_{q}(\operatorname{SL}(2))$-module with basis $A, B, C, I$ and action of $H$ given by

$$
\begin{array}{llll}
A \cdot a=A+I, & A \cdot d=A-I, & A \cdot b=0 & A \cdot c=0, \\
B \cdot a=q^{-1} B, & B \cdot d=q B, & B \cdot b=I, & B \cdot c=0, \\
C \cdot a=q^{-1} C, & C \cdot d=q C, & C \cdot c=I, & C \cdot b=0,
\end{array}
$$

and

$$
I \cdot x=\varepsilon(x) I \quad \text { for all } x \in H .
$$

Using the lemma we obtain a module algebra structure on the factor algebra $E$ of $T(V)$ by the ideal generated by the following elements (we omit the tensor signs):

$$
\begin{aligned}
& A B-B A-2 B, \quad A C-C A+2 C, \\
& B C-q^{2} C B-1 /\left(1-q^{-2}\right), \quad I-1 .
\end{aligned}
$$

Next we observe that the algebra $E$ may be constructed as an iterated skew polynomial ring

$$
F[A] \subseteq F[A, B] \subseteq F[A, B, C]=E
$$



0 .

It follows that $E$ has a basis consisting of monomials $A^{i} B^{j} C^{k}$ with $i, j, k \geq$

Theorem. As a right $H$-module, $E \cong E_{H}(H / m)$. However, $E$ is not isomorphic to a submodule algebra of $H^{0}$.

Proof. Since $I=c H$ is a bi-ideal in $H, E^{\prime}=\operatorname{ann}_{E} I$ is a submodule algebra of $E$. We claim that $E^{\prime}=F[A, B]$. Since $A \cdot c=B \cdot c=0$ and $E^{\prime}$ is a subalgebra, we see that $F[A, B] \subseteq E^{\prime}$. To prove the reverse inclusion first note that for $f \in F[A, B]$ we have $f=f \cdot\left(a d-q^{-1} b c\right)=f \cdot a d$ and $f \cdot$ $(a d-d a)=f \cdot\left(q^{-1}-q\right) b c=0$, so $f=f \cdot a d=f \cdot d a$. Now if $e \in E$, we can write $e=\sum_{n=0}^{m} e_{n} C^{n}$ with $e_{n} \in F[A, B]$. An easy induction shows that $C^{n} \cdot c=\left(q^{n}-q^{-n}\right) /\left(q-q^{-1}\right) C^{n-1}$. Thus since $F[A, B] \cdot c=0$, we obtain

$$
\begin{aligned}
e \cdot c & =\sum\left(e_{n} \cdot c\right)\left(C^{n} \cdot a\right)+\left(e_{n} \cdot d\right)\left(C^{n} \cdot c\right) \\
& =\sum\left(q^{n}-q^{-n}\right) /\left(q-q^{-1}\right)\left(e_{n} \cdot d\right) C^{n-1} .
\end{aligned}
$$

Hence, if $e_{m} \neq 0$ and $m \geq 1$, then $e_{m} \cdot d \neq 0$, since $e_{m} \cdot d a=e_{m}$, so $e \cdot c \neq 0$. Therefore, $E^{\prime}=F[A, B]$.

Next we observe that $E^{\prime}=F[A, B]$ is the injective hull of $H / m$ as an $\left(H / I \cong O_{q}(P)\right)$-module. This follows by comparing the action defined above with that in $\S 3.3$ (replacing $x, y, X, Y_{1}$ in $\S 3.3$ by $d, b,-A$, and $B$, respectively).

Now note that if $e=\sum_{n=0}^{m} e_{n} C^{n} \in E$, with $e_{m} \neq 0$, then by $(*) e \cdot c^{m}$ is a nonzero element of $E^{\prime}$. Hence, $E$ is an essential extension of $E^{\prime}$ and therefore also of $H / m$. By the Theorem in $\S 2.1$, to show that $E \cong E_{H}(H / m)$ it is enough to show that $E \cdot c=E$. By $(*)$ we see that $E \cdot c$ contains $(F[A, B] \cdot d) C^{n}$ for all $n$, but $F[A, B] \cdot d=F[A, B]$ since $f=f \cdot a d$ for all $f \in F[A, B]$. This shows that $E \cong E_{H}(H / m)$.

Finally to show that $E$ is not isomorphic to a submodule algebra of $H^{0}$ suppose that $A^{\prime}, B^{\prime}, C^{\prime}$ are elements of $H^{0}$ such that

$$
A^{\prime} B^{\prime}-B^{\prime} A^{\prime}=2 B^{\prime}, \quad A^{\prime} C^{\prime}-C^{\prime} A^{\prime}=-2 C^{\prime}
$$

and

$$
B^{\prime} C^{\prime}-q^{2} C^{\prime} B^{\prime}=1 /\left(1-q^{-2}\right) .
$$

Then $2\left\langle B^{\prime} \mid 1\right\rangle=\left\langle A^{\prime} \mid 1\right\rangle\left\langle B^{\prime} \mid 1\right\rangle-\left\langle B^{\prime} \mid 1\right\rangle\left\langle A^{\prime} \mid 1\right\rangle$ gives $\left\langle B^{\prime} \mid 1\right\rangle=0$ and similarly $\left\langle C^{\prime} \mid 1\right\rangle=0$. From the last equation we obtain the contradiction $0=1 /\left(1-q^{-2}\right)$.

3.5. Remark. In $\S 3.4$ it does not seem possible to construct $E$ using the Corollary in $\S 1.1$. Note the ideal $I$ is generated by a normal element of $H$, but this ideal is not complemented by a subalgebra of $H$. On the other hand, the ideal $m$, although complemented by the subalgebra $F$, does not have the AR property.

\section{REFERENCES}

[A] E. Abe, Hopf algebras, Cambridge Univ. Press, Cambridge, 1977.

[APW] H. H. Andersen, P. Polo, and K. Wen, Representations of quantum algebras, Invent. Math. 104 (1991), 1-59.

[CM] W. Chin and I. M. Musson, Hopf algebra duality, injective modules and quantum groups, Communications in Algebra (to appear). 
[D] V. G. Drinfeld, Quantum groups, Proc. Internat. Congr. Math., Berkeley, CA, 1987, pp. 798820.

[G] J. A. Green, Locally finite representations, J. Algebra 41 (1976), 137-171.

[H] R. Hartshorne, Algebraic geometry, Springer, New York, 1977.

[K] T. G. Kucera, Explicit descriptions of injective envelopes: generalizations of a result of Northcott, Comm. Algebra 17 (1989), 2703-2715.

[HL] T. J. Hodges and T. Levasseur, Primitive ideals in $C_{q}[G]$, University of Cincinnati, preprint.

[L] T. Levasseur, L'enveloppe injective du module trivial sur une algebre de Lie resoluble, Bull. Sci. Math. (2) 110 (1986), 49-61.

[Ma] Y. I. Manin, Quantum groups and non-commutative geometry, Centre de Recherches Math., Montreal, 1988.

[McR] J. C. McConnell and J. C. Robson, Noncommutative Noetherian rings, Wiley, Chichester, 1987.

[M] I. M. Musson, On the structure of certain injective modules over group algebras of soluble groups of finite rank, J. Algebra 85 (1983), 51-75.

[N] D. G. Northcott, Injective modules and inverse polynomials, J. London Math. Soc. (2) 8 (1974), 290-296.

[R] D. E. Radford, Generalized double crossproducts associated with the quantized enveloping algebras, University of Illinois at Chicago, preprint.

[SV] D. W. Sharpe and P. Vamos, Injective modules, Cambridge Univ. Press, Cambridge, 1972.

[T] M. Takeuchi, Hopf algebra techniques applied to the quantum group $U_{q}(\mathbf{s l}(2))$, Deformation Theory and Quantum Groups with Applications to Mathematical Physics (J. Stasheff and M Gerstenhaber, eds.), Contemp. Math., vol. 134, Amer. Math. Soc., Providence, RI, 1992, pp. 309-323.

Department of Mathematical Sciences, University of Wisconsin, P.O. Box, 413, MilWAUKEE, WISCONSIN 53201-0413

E-mail address: musson@csd4.csd.uwm.edu 